OPEN ACCESS

Edited by:

Laura N. Vandenberg, University of Massachusetts Amherst, United States

Reviewed by: Atsuto Onoda,

Sanyo-Onoda City University, Japan Jennifer Joan Adibi, University of Pittsburgh, United States

*Correspondence: Sean M. Richards seanrichards.utc@gmail.com

Specialty section: This article was submitted to Developmental and Reproductive Toxicology, a section of the journa Frontiers in Toxicology

Received: 29 June 2021 Accepted: 29 October 2021 Published: 22 November 2021

Citation:

Chambers WS, Hopkins JG and Richards SM (2021) A Review of Perand Polyfluorinated Alkyl Substance Impairment of Reproduction. Front. Toxicology 3:732436. doi: 10.3389/ftox.2021.732436

\section{A Review of Per- and Polyfluorinated Alkyl Substance Impairment of Reproduction}

\author{
Weston S. Chambers ${ }^{1}$, Jaida G. Hopkins ${ }^{1}$ and Sean M. Richards ${ }^{1,2 *}$ \\ ${ }^{1}$ Department of Biology, Geology and Environmental Sciences, University of Tennessee at Chattanooga, Chattanooga, TN, \\ United States, ${ }^{2}$ Department of Obstetrics and Gynecology, Section on Maternal-Fetal Medicine, University of Tennessee College \\ of Medicine, Chattanooga, TN, United States
}

In this review article, we compiled peer-reviewed literature describing PFAS exposure and reproductive effects in animals and humans. The aim was to compare environmental occurrence and effects of the most prominent long-chain PFAS compounds and their short-chain replacements. Long-chain PFAS compounds are known to persist in the environment due to their chemical stability, and also known to bioaccumulate; hence, these compounds are being replaced globally. Indeed, PFOA and PFOS are considered long-chain "forever pollutants," and thus the potential reproductive risk may continue for decades. Much less is known about their short-chain replacements despite the fact that they becoming more widespread in the environment. Short-chain PFAS are generally less bioaccumulative than long-chain, but they are more mobile and persistent in aquatic ecosystems. The three most prominent of these are commonly referred to as GenX, ADONA and F53B. The short-chain PFAS have similar physical and chemical properties as their predecessors; however, because they are relatively new, much less is known about the potential to disrupt reproduction. Indeed, high-quality epidemiological studies are needed to determine associations between short-chain PFAS exposure and effects on reproductive health. However, epidemiological evidence is mounting that long-chain PFAS exposure is associated with reproductive effects (i.e., decrease in fertility, reduced fetal growth and birth weight, pregnancy-induced hypertension and preeclampsia, thyroid hormone disruption during pregnancy, and preterm birth). Evidence from animal models and human cell lines indicates that short-chain PFAS similarly affect reproductive endpoints; however, epidemiological studies are scarce and inconsistent. Although short-chain PFAS have been quantified in drinking water and sediment worldwide, most of these studies did not focus on quantitation of GenX, ADONA, and F53B. There are also many other short-chain PFAS byproducts of manufacturing that have yet to be identified and studied. When sum total concentration of long- and short-chain PFAS are considered, the concentration rises by an order or magnitude or greater, as will the risk of exposure and subsequent reproductive effects.

Keywords: PFAS, reproduction impairment, short-chain PFAS, GenX, ADONA, F53B 


\section{INTRODUCTION}

\section{Long-Chain PFAS}

Per- and Polyfluorinated Alkyl Substances (PFAS) are synthetic chemicals that contain at least one perfluoroalkyl group (Birru et al., 2021). Perfluorooctanoic acid (PFOA) and perfluorooctane sulfonate (PFOS) are the most common PFAS detected in the environment due to their widespread use in manufacturing and chemical stability (Huang and Jaffé, 2019). Both PFOS and PFOA are considered long-chain PFAS because they possess an eightcarbon backbone; their functional groups are sulfonate and carboxylate, respectively (Tsuda, 2016). They possess both hydrophobic and oleophobic properties, along with other chemical characteristics making them useful in many consumer goods (Birru et al., 2021). Their carbon-fluorine bonds, make them resistant to degradation and allow them to persist in the environment and bioaccumulate within living organisms (Zeng et al., 2019; Neagu et al., 2021).

After more than 50 years of production, PFOS and PFOA were widely discovered in humans and the environment, and a subsequent phasing out of production by the $3 \mathrm{M}$ company began in 2000 (Giesy and Kannan, 2001; Olsen et al., 2017). Subsequently, the United States Environmental Protection Agency procured an agreement with other major manufacturing companies to reduce long-chain PFAS emissions; other global environmental regulations for PFAS emerged as well (Olsen et al., 2017). The phasing out of these compounds resulted in a decline in human blood serum concentrations of PFOS and PFOA between the years of 2000 and 2015 (Olsen et al., 2017; Wang et al., 2017). Despite the reduction in production of long-chain PFAS, the compounds persist in the environment and in humans and are a continuing concern due to their widespread environmental persistence, distribution, potential toxicological effects, potential to bioaccumulate (Huang and Jaffé, 2019).

Ingestion is considered the largest exposure route to humans (rather than dermal and inhalation). Tap water is a major PFAS exposure route where most average PFAS concentrations are in the low ng/L range (Sinclair et al., 2020). Regarding PFOA and PFOS, the USEPA has issued a Health Advisory Level of $70 \mathrm{ppt}$ and is in the process of making a final regulatory determination (USEPA, 2021). Seafood is a significant PFAS exposure route while exposure from consumer goods, food packaging, and indoor environments are uncertain (Sunderland et al., 2019).

\section{Short-Chain PFAS Replacements}

With the phasing out of long-chain PFAS in the early 2000s, short-chain alternatives (i.e., those with carbon backbones of $<7$ carbons) began to take their place in industry and the environment (Brendel et al., 2018). These alternatives share similar structures to long-chain PFAS (i.e., heavily fluorinated carbon chains), however, one or more alkylether group is inserted into a shorter fluoroalkyl chain. Short-chain PFAS are also products of long-chain degradation (Li et al., 2020). Shortchain replacements GenX, ADONA, and F53B are widely used. GenX is a trade name for a short-chain PFAS processing technology, of which the hexafluoropropylene oxide (HFPO) dimer acid and its ammonium salt are the major constituents. ADONA (dodecafluoro-3H-4,8-dioxanonanoate) is generally used as a substitute for PFOA in fluoropolymer production (Fromme et al., 2017; Munoz et al., 2019). Chlorinated polyfluoroalkyl ether sulfonate, or F53B, has been produced as an alternative to PFOS and has been adopted as a mist suppressant by several electroplating companies (Du et al., 2016; Munoz et al., 2019; Shi et al., 2019).

\section{ENVIRONMENTAL CONCENTRATIONS}

\section{Long-Chain PFAS}

Water is the major environmental sink for long- and short-chain PFAS. Bai and Son (2021) quantified concentrations of 17 PFAS in the Las Vegas and Reno watersheds (Nevada, USA), and compared them to data on aquatic PFAS concentrations worldwide. Overall, Bai and Son (2021) found that concentrations of individual PFAS ranged from non-detectable to low ppt (maximum of $74.47 \mathrm{ng} / \mathrm{L}$ ) which was similar to studies and findings in watersheds of the United States (Nakayama et al., 2007; Zhang et al., 2016), Europe (Moller et al., 2010), Uganda (Dalahmeh et al., 2018), China (So et al., 2007; Zhao et al., 2013; Wang et al., 2019), and Australia (Clara et al., 2009). In surface waters where sewage effluent is the primary input, maximum PFAS concentrations were $207.59 \mathrm{ng} / \mathrm{L}$; groundwater contaminated with landfill leachate maximum PFAS concentrations were 5,200 ng/L (Zhou W. et al., 2017). PFAS have been detected in the tissues of many wild species, including, but not limited to, bald eagles, albatrosses, polar bears, seals, dolphins, alligators, squid, and many fish species (Giesy and Kannan, 2001; McCarthy et al., 2017).

\section{Short-Chain PFAS}

Although atmospheric deposition is a possibility, the main source of GenX and ADONA in surface water is manufacturer's wastewater (Hopkins et al., 2018). F53B is primarily released into waterways via chrome plating industries, finding its way into nearby irrigation systems and ground water. The shorter length and alkylether substitution of short-chain PFAS contribute to a greater water solubility. GenX, when in water, loses an ammonium group, creating an anion that is the same as the HFPO-DA anion in water (Hopkins et al., 2018). This anion has a precursor that travels via air, the $\mathrm{C} 3$ dimer acid fluoride (Hopkins et al., 2018). Once the $\mathrm{C} 3$ dimer acid fluoride interacts with water, it creates the same anion which readily migrates to groundwater, contributing to private well contamination (Hopkins et al., 2018). The limited data indicate that most short-chain PFAS are more persistent and widespread than long-chain PFAS in the aquatic environment (Olsen et al., 2017; Wang et al., 2017; Brendel et al., 2018; Li et al., 2020). GenX chemicals are present in multiple aquatic environments including surface water, groundwater, and in drinking water and rainwater (USEPA, 2018). Aside from factory wastewater effluent or points very close to industry outfalls, concentrations of GenX, ADONA, and F53B are found in surface waters in the low ng/L concentrations (Pan et al., 2018; Munoz et al., 2019; Gebbink and van Leeuwen, 2020; 
Bai and Son, 2021). Both GenX and F53B were found in higher concentrations than long-chain PFAS in surface water samples collected downstream from a PFAS manufacturer (Gebbink et al., 2017; Ateia et al., 2019). And although short-chain PFAS have been quantified in drinking water and sediment worldwide, most studies did not focus on quantitation of GenX, ADONA, and F53B and thus, data in the aquatic environment are lacking (Ateia et al., 2019).

Bai and Son (2021) found that short-chain PFAS were more likely to partition in the water column, while long-chain PFAS most likely to partition in sediment. This is similar to the finding of Zhao et al. (2016) who estimated that $88.8 \%$ of total PFAS in the water column are short-chain. These estimations correspond to what would be expected based on the greater water solubility of short-chain PFAS.

It is important to note that the studies that concluded "relatively low" concentrations of PFAS in the water column or sediment are often describing the concentration of individual molecules. While environmental regulations limit concentrations of individual PFAS, the sum of the thousands of PFAS need to be considered for estimations of exposure and effects. Indeed, even if toxicity is considered to be simply additive, estimations of risk must include total PFAS exposure.

\section{PFAS PRESENCE IN HUMANS}

Routes of human exposure to PFAS include food, drinking water, house dust, ambient and indoor air, and consumer products (Olsen et al., 2017). Examples of consumer products that have utilized PFAS in the past include fabrics, carpet, grease-proof food-contact paper, nonstick cookware, paints, and cosmetics (Kjeldsen and Bonefeld-Jørgensen, 2013; Olsen et al., 2017).

For populations close to sources of contaminated water sources, drinking water is the dominant exposure pathway (Vestergren and Cousins, 2009). This was illustrated by Fromme et al. (2017); blood plasma samples were tested for the presence of PFOS, PFOA, and ADONA in humans living within an $80 \mathrm{~km}$ radius of a former PFOA production plant, where ADONA was being used as a replacement emulsifier. Drinking water was the known exposure route. The 95th percentile concentrations in blood plasma were $13.5 \mu \mathrm{g} / \mathrm{L}$ and $85.5 \mu \mathrm{g} / \mathrm{L}$ for PFOS and PFOA, respectively. Blood plasma concentrations of ADONA were just above detection levels $(0.2 \mu \mathrm{g} / \mathrm{L})$, indicating that health risks were not likely (Fromme et al., 2017).

Food is also a major source of exposure, PFAS have been detected in fish fillets, blood serum of beef cattle, fruits and vegetables, bread, and milk (Vestergren and Cousins, 2009). Human breast milk is a source of infant exposure to PFAS, and these compounds have been detected in samples in the United States, Asia, and Europe (Tao et al., 2008). PFAS have also been found in human umbilical cord blood samples, with one study detecting trace amounts in $100 \%$ of tested samples (Apelberg et al., 2007). These findings, indicating an exposure pathway in utero, are concerning due to the high vulnerability to toxicants during fetal development (Bach et al., 2014).
PFOS and PFOA are readily absorbed in the gastrointestinal tract, excreted in urine and feces, and do not undergo metabolism. Estimated human half-lives for PFOS and PFOA are approximately 5 years and 2-4 years, respectively (EFSA, 2018). PFAS bind to blood serum proteins, allowing them to travel through the circulatory system and subsequently accumulate in most organs in the human body, particularly in the kidneys, liver, spleen, brain, and testicles (Jensen and Leffers, 2008; Wang et al., 2021). Elimination of PFAS from the body is done via renal clearance. Clearance varies widely by species. In humans it is a relatively slow process with half-lives of 3.8 years for PFOA and 5.4 years for PFOS (Behr et al., 2018). Much shorter half-lives are observed in other animals, with male mice, rabbits, chickens, and cynomolgus monkeys having half-lives of $12,0.23,4.6$, and 30 days, respectively (Vestergren and Cousins, 2009). For water-breathing organisms, the high aqueous solubility allows for easier elimination of the compounds through the gills (Kelly et al., 2009).

\section{BIOACCUMULATION}

Another reason for the phasing out of long-chain PFAS is the potential of these compounds to bioaccumulate (Olsen et al., 2017). In Lake Ontario, the concentrations of long-chain PFAS were higher within the biota and sediment than the water, giving a bioaccumulation factor between lake trout and water of $3.4 \times$ $10^{4} \mathrm{~L} / \mathrm{kg}$ (Houde et al., 2008). Zooplankton contained $195 \mathrm{ng} / \mathrm{g}$, sediment contained $187 \mathrm{ng} / \mathrm{g}$, and the water contained $2.2 \mathrm{ng} / \mathrm{L}$ (Houde et al., 2008). Some predatory species have been observed to contain higher levels of PFAS than in their diets, indicating that PFAS can biomagnify in higher trophic levels (Giesy and Kannan, 2001). Biomagnification is seen only in non-aquatic predators, such as seals and polar bears, most likely due to the proteinophilic nature of PFAS, relatively high aqueous solubility of PFAS, and the low volatility (Kelly et al., 2009).

Because of the increased water solubility of short-chain PFAS, most studies indicated a lower bioaccumulation potential. Indeed, short-chain PFAS are eliminated from the body quicker, and therefore have less potential to bioaccumulate (Gomis et al., 2018). Carbon-chain length is generally inversely proportional with elimination rates; this trend follows for humans as well (Lau, 2012). For example, a four carbon PFAS, perfluorobutanoate (PFBA), had a measured half-life of 3 days in humans, which is a stark difference from the years-long half-lives of PFOS and PFOA (Lau, 2012). While short-chain PFAS may not remain in biota for extended periods of time, this does not necessarily indicate that they have less potential toxicity than long-chain PFAS (Gomis et al., 2018). Short-chain PFAS are still very persistent in the environment, indicating that there is potential for high environmental exposure (Gomis et al., 2018). However, the faster rate of clearance of short-chain PFAS could potentially reduce developmental toxicity (Lau, 2012). Also, there is no current evidence that biotransformation of these chemicals takes place within the body due to their structural stability, however, precursors to these molecules have exhibited biotransformation into perfluoroalkyl acids (Sunderland et al., 2019). 
While it is generally accepted that short-chain PFAS bioaccumulate less than long-chain PFAS, the bioaccumulation of ADONA and GenX is not well researched (Brendel et al., 2018; Munoz et al., 2019; Fan et al., 2020). To further complicate the issue of short-chain PFAS bioaccumulation, there is some evidence that F53B may biomagnify in aquatic food webs (Munoz et al., 2019). To address this data gap, integrative computational approaches are being used to estimate PFAS bioaccumulation effects across several species. This approach found a pattern of similar binding affinities to liver fatty acidbinding protein across nine different species, including humans, chickens, and rainbow trout (Cheng et al., 2021). This approach indicates similar PFAS bioaccumulation potentials across species and illustrates how computational approaches can be used to estimate binding to other receptors and ligands. However, the bioaccumulation potential of short-chain PFAS needs to be further researched in live animal models.

\section{PFAS REPRODUCTIVE EFFECTS}

\section{Long-Chain Reproductive Effects in Animal Models}

Researchers have utilized many different model organisms in order to better understand the reproductive effects of PFOS on living organisms. Shi et al. (2008) observed zebrafish embryos exposed $4 \mathrm{~h}$ post-fertilization to varying concentrations of PFOS between 0.1 and $5 \mathrm{mg} / \mathrm{L}$, and observed significant changes in embryo development. Noted changes included delayed hatching, malformation, and depressed heart rates, which led to reduced survival rates of fry exposed to $1 \mathrm{mg} / \mathrm{L}$ PFOS for 21 days before hatching (Shi et al., 2008). Han and Fang conducted a study to observe the effects of PFOS exposure on swordtail fish; they found a significant decrease in survival rate of offspring at higher levels of PFOS exposure in these swordtail fish (Han and Fang, 2010). PFOS exposure has been associated with inhibition of sperm production (Qu et al., 2016). In female mice, PFOS exposure is associated with ovulation reduction (Wang et al., 2018). Exposure of pregnant mice to PFOS resulted in neonatal mortality as a result of severe lung collapse and intracranial blood vessel dilation (Yahia et al., 2010).

While PFOA is similar to PFOS in structure, PFOS is generally considered to be more toxic (Zheng et al., 2012). Similar research has been conducted on PFOA as that on PFOS in order to understand the reproductive effects of the compound. A study was conducted to examine the effects of PFOA on development of zebrafish and found that PFOA is acutely toxic to zebrafish embryos $\left(\mathrm{LC}_{50}=262 \mathrm{mg} / \mathrm{L}\right.$ ), similar to PFOS (Zheng et al., 2012). At higher concentrations, edema, delayed hatching, and spinal malformations were observed, and nearly $100 \%$ mortality was observed when the embryos were exposed to $270 \mathrm{mg} / \mathrm{L}$ (Zheng et al., 2012). A meta-analysis was conducted to determine the effects of PFOA on the reproductive system of male rats and found significant association between higher PFOA levels and reproductive toxicity (Wang et al., 2021). Toxicological effects of increased serum PFOA levels included a decrease in serum testosterone levels and a decrease in weight of reproductive organs (testicle and epididymis) (Wang et al., 2021). The exposure of PFOA to pregnant mice resulted in reduced fetal body weight and delayed bone formation, and a 100\% mortality rate of pups after exposure at $10 \mathrm{mg} / \mathrm{kg}$ was observed (Yahia et al., 2010). PFOA increased mortality of the pups, similarly to PFOS, but no intracranial blood vessel dilation was observed, indicating a different cause of death by PFOA (Yahia et al., 2010).

PFAS interact directly with estrogen receptor $\alpha(E R \alpha)$ in trout liver in vivo, although they have a weak affinity and they are noted to induce an estrogenic effect (Benninghoff et al., 2010). In male mice, PFOS exposure was observed to down-regulate ERa and up-regulate estrogen receptor $\beta$, which increased cell apoptosis and decreased cell proliferation in the testes (Qu et al., 2016). In female mice, PFOS was shown to suppress ERa receptors, which led to induced prolongation of diestrus and ovulation reduction (Wang et al., 2018). Accordingly, Jensen and Leffers (2008) found that male rats exposed to PFOA developed Leydig cell hyperplasia eventually resulting in adenomas (Jensen and Leffers, 2008). A decrease in testosterone production was also reported in these rats followed by an increase in estradiol levels.

Reproductive functions are dependent on peroxisome proliferator-activated receptors (PPARs). These receptors are susceptible to PFAS which are known to affect gene transcription through the activation of PPARs (Vitti et al., 2016). When mice were treated with PFOA, subsequent changes in PPARs were associated with delayed mammary gland development (Zhoa et al., 2012).

\section{Short-Chain Reproductive Effects in Animal Models}

Three recent studies examined reproductive effects of GenX in mice (Blake et al., 2020) and rats (Conley et al., 2019; Conley et al., 2021). Mice treated with GenX had a greater incidence of placental abnormalities while rats showed a greater incidence of reduced maternal thyroid hormone levels and elevated levels of PPAR-regulated gene expression in both maternal and fetal livers (as with PFOA described above). GenX is a developmental toxicant in the rat; those dosed from gestational day 8 through post-natal day 2 (from 1 to $125 \mathrm{mg} / \mathrm{kg} /$ day), had increased neonatal mortality and reduced birth weight (Conley et al., 2021).

In zebra fish, F53B was associated with an increase in birth defects, delayed hatchings, and decreased survival rates in embryos (Shi et al., 2017). Conversely, Gaballah et al. (2020) concluded that GenX (and ADONA) was not associated with developmental toxicity in zebrafish.

\section{Long-Chain Reproductive Effects in Human Cell Lines}

There is conflicting evidence regarding whether or not PFAS interact with estrogen receptors in humans. Multiple studies have concluded that PFAS promote activity of estrogen receptors in the presence of estradiol, but do not directly interact with them (Kang et al., 2016; Behr et al., 2018). Another study found that PFAS do not have any effect on ER, while another found that PFAS bind directly to ER without the presence of estradiol (Kjeldsen and 
Bonefeld-Jørgensen, 2013; Yao et al., 2014). Despite being heavily researched, a lack of understanding of the mode of action of these pollutants persists (Cousins et al., 2020).

Zhang et al. (2015) exposed human syncytiotrophoblasts isolated from term placenta to PFOS. Dose-dependent reductions of human chorionic gonadotropin (hCG), estradiol, and progesterone resulted. After blocking the apoptotic route, the authors concluded that PFOS exerted its effects by inducing apoptosis due (Zhang et al., 2015).

\section{EPIDEMIOLOGICAL STUDIES OF PFAS}

\section{Long-Chain PFAS}

Multiple epidemiological studies have been conducted to determine the associated effects of PFAS on humans. Johnson et al. (2014) found an association between lower birth weights and exposure to PFOA, noting an $18.9 \mathrm{~g}$ decrease in birth weight for every $\mathrm{ng} / \mathrm{mL}$ increase in serum PFOA level. As described in rat models above (Jensen and Leffers, 2008), Leydig cell hyperplasia is connected with lower testosterone levels in men, indicating a possible mode of action for PFOA to induce testicular cancer (Tarapore and Ouyang, 2021).

As also described above, the effects of PFAS on human reproductive hormones is uncertain. One epidemiological study found that an increase in human serum PFAS levels was negatively associated with serum levels of reproductive hormones, including testosterone (Tsai et al., 2015). However, another study found a positive correlation between increased PFAS levels and levels of testosterone and estradiol, while yet another study found no correlation between PFAS and sex hormone levels (Olsen et al., 1998; Sakr et al., 2007). PFAS have also been shown to compete with thyroxine to bind to transthyretin (Kar et al., 2017). Both molecules play a major role in thyroid function (Kar et al., 2017). Luo et al. (2021) recruited 902 men for a cross-sectional study of 24 PFAS compounds in blood plasma. Five reproductive hormones: total testosterone (TT), $\mathrm{E}_{2}, \mathrm{FSH}, \mathrm{LH}$, and insulin like factor 3 (INSL3), and SHBG were measured. They found a significant inverse relationship between PFAS mixture with $\mathrm{E}_{2}$ and $\mathrm{E}_{2} / \mathrm{TT}$. Xie et al. (2021) studied a large cohort of males and females aged from 12 to 80 and found that PFAS exposure was associated with alterations in sex hormones (including TT, free testosterone, $\mathrm{E}_{2}$, and $\mathrm{SHBG}$ ) in a sex-, age-, and compound-specific manner. Multiple epidemiologic studies (Taylor et al., 2014; Barrett et al., 2015; Zhou Y. et al., 2017) associate PFAS exposure with effects indicating ovarian alterations (e.g., altered menstrual cycle, hormone alterations, early menopause). Petersen et al. (2018) examined PCBs and PFAS blood concentrations and associated effects on semen quality and reproductive hormones in Faroese men. Concentrations of total PCBs and PFOS were positively associated with sex hormone-binding globulin (SHBG) and luteinizing hormone (LH). While the PCB concentrations in the Faroese men were relatively high (compared to men around the world), the PFAS concentrations were relatively similar. Overall, the authors concluded that the positive association to $\mathrm{LH}$ for both $\mathrm{PCBs}$ and PFOS indicate a direct toxic effect on Leydig cells.

Petersen et al. (2020) qualitatively assessed 26 epidemiological studies for evidence of associations between PFAS exposures and male reproductive health. Several studies found some evidence that single PFAS compounds were associated with specific male reproductive parameters (e.g., semen quality, reproductive hormones, cryptorchidism, hypospadias, and testicular cancer). However, the overall conclusions were limited due to a lack of consistency between studies.

\section{Short-Chain PFAS}

Data for short-chain PFAS effects on human reproductive parameters are scarce and these data are inconsistent (Nian et al., 2020; Brase et al., 2021). Indeed, the threat that these long-chain replacements pose on human populations, pathophysiology and health effects are not completely understood (Sunderland et al., 2019). Effects in humans are difficult to extrapolate from laboratory dose-response results. However, existing data indicates that concentrations in humans are lower than dose-response effects found in the laboratory. For example, So et al. (2007) found a maximum human PFAS concentration of $360 \mathrm{ng} / \mathrm{L}$ (breast milk), orders of magnitude below laboratory animal model exposures which induced toxicity.

Because of the likelihood of co-occurrence, short- and longchain PFAS have been studied together. A three year study of 752 women in China found prenatal exposure to one short-chain and one long-chain PFAS (perfluorobutanesulfonate, PFBS and perfluoroheptanoic acid, $\mathrm{PFHpA}$, respectively) was associated with alterations of fetal gonadotropins as well as free androgen levels (Nian et al., 2020). A recent review of endocrine disruption effects associated with both long- and short-chain PFAS exposure found that effects varied similarly according to gender and age of development; in some cases, short-chain effects were greater than long-chain PFAS (Mokra, 2021). While much more is needed pertaining to human reproductive effects, the implications of these studies warrant further epidemiological studies on shortchain PFAS.

\section{DATA GAPS AND LIMITATIONS}

There are similar data gaps and limitations between wildlife and human PFAS exposure reproductive effects. Statistically representative population surveys are lacking for assessing PFAS exposure in wildlife (DeSilva et al., 2021). Laboratory, field, and epidemiological studies are needed to effectively assess effects and risk of PFAS compounds in singular exposure and mixtures. However, because almost 20 years of data exist for long-chain PFAS, there is a considerably greater dearth of data on next-generation PFAS as well as next-gen precursors and degradation products. Mixture toxicity data for all PFAS are woefully inadequate which further complicates our understanding of potential sub-lethal and chronic effects. Molecular markers or metabolic fingerprints for PFAS exposure and effects are needed to effectively develop effective 
PFAS regulations (Sinclair et al., 2020). Petersen et al. (2020) cite a glaring knowledge gap regarding exposures prior to adulthood, exposure to mixtures of PFAS compounds and other potential endocrine disruptors. Epidemiological studies conducted to date are limited in the conclusions and comparisons that can be made because of inconsistency between studies. High-quality epidemiological studies are needed to determine associations between PFAS exposure and effects on male reproductive health (Petersen et al., 2020).

Data on the effects of PFAS at environmentally-relevant concentrations are limited. This hampers the effective management of these compounds.

There is some evidence that toxicity detected in laboratory animal models and human cell lines occurs at higher PFAS concentrations than what occurs in the environment. While there is some evidence to support this from the HQ perspective, there are significant limitations to the HQ approach. Because the HQ method is based on LC50 values, it tends to neglect the subtle non-lethal effects associated with reproduction. In addition, the dramatically different half-lives described herein further complicates blanket statements about PFAS risk across multiple species. Indeed, the HQ approach could underestimate non-lethal effects and subsequent effects in reproduction of wildlife and humans alike.

Despite HQ values < 1 , many epidemiological assessments of PFAS exposure indicate correlations with non-lethal reproductive effects. The USEPA cited concerns about effects on reproductive parameter in their recent drinking water health advisory for PFOA and PFOS (USEPA, 2021). Given the similarities of short-chain PFAS to these compounds, concern for effects at environmentally-relevant concentrations is warranted.

Phospholipids and proteins affect tissue partitioning and accumulation of PFAS differently than other long-lived, bioaccumulative toxicants. More research on PFAS mode of action is needed (e.g., endocrine disruption) as well as differences in PFAS toxicokinetic properties between compounds. Indeed, the unique toxicokinetics of PFAS, make standard bioaccumulation metrics unsuitable. Improved PFAS bioaccumulation models are being developed which should lead to more accurate risk assessments in wildlife and humans (DeSilva et al., 2021). This is imperative to understand differing responses and half-lives between the sexes and among species and life stages (Fenton et al., 2021).

Most PFAS research has been conducted on but a few prominent compounds with numerous associated reproductive health impairments. There are thousands of PFAS compounds cycling through the environment and industry for which toxicity data are non-existent. To adequately address this enormous issue more contemporary and high-throughput approaches such as read-across, molecular dynamics, and protein modeling are needed (Fenton et al., 2021).

Improvements are needed in human modelling of exposure, focusing on PFAS exposure susceptibility windows and statistical modeling of data to account for endocrine disruption (Tarapore and Ouyang, 2021). However, care must be taken to address mixture issues, and properly attribute toxic effects and mechanisms to a specific PFAS or mixture of PFAS.

To properly estimate exposure potential, data on environmental concentrations need to be integrated to develop mass balance models of PFAS in different matrices (e.g., land, water, air), hydrologic sub-divisions, and to track the migration pathway of short-chain PFAS to drinking water (Ateia et al., 2017). Short-chain PFAS are resistant to conventional municipal surface water purification methods (Ateia et al., 2019). While drinking water purification trials using granular activated carbon have reported a removal efficiency of $99 \%$ (Du et al., 2016), largescale, affordable municipal drinking water methods are not widely available.

\section{CONCLUSION}

The chemical properties of PFAS are valuable for manufacturing and consumer convenience; however, these same properties make them resistant to degradation and persistent in the environment, wildlife, and humans. Generally, longer carbon chains in PFAS correlate with higher toxicity, however, differential reproductive effects are observed in vitro and in vivo. Short-chain PFAS tend to bioaccumulate less, but are more persistent in surface water. While surface water data show individual short-chain PFAS presence to be in the low ng/L range, sum total concentrations of PFAS are orders of magnitude higher. Some studies indicate that reproductive effects of PFAS seen in laboratory models and human cell lines are at concentrations greater than what will be realized in the environment. Most studies do not consider mixture toxicity; epidemiological studies inherently address mixture toxicity. Epidemiological studies indicate that environmentally-relevant PFAS concentrations are associated with reproductive effects. Indeed, even though most ecological and epidemiological studies quantify a few PFAS, these studies are effectively assessing the impact of many more PFAS. The majority of evidence for reproductive effects is linked to longchain PFAS exposure. Long-chain PFAS is on the decline worldwide but will remain an exposure issue. Short-chain PFAS exposure is on the rise. Even though some specific short-chain PFAS have been studied, data on reproductive effects of short-chain PFAS are considerably less than for long-chain. In addition, there are many short-chain PFAS byproducts from manufacturing that have yet to be identified.

\section{AUTHOR CONTRIBUTIONS}

$\mathrm{WC}$ is the primary author, having written a majority of the initial draft. JH is a contributing author, having written a portion of the initial draft. SR is the corresponding and senior author, developed the outline, guided the development of the manuscript, and handled all revisions and edits. 


\section{REFERENCES}

Apelberg, B. J., Witter, F. R., Herbstman, J. B., Calafat, A. M., Halden, R. U., Needham, L. L., et al. (2007). Cord Serum Concentrations of Perfluorooctane Sulfonate (PFOS) and Perfluorooctanoate (PFOA) in Relation to Weight and Size at Birth. Environ. Health Perspect. 115, 1670-1676. doi:10.1289/ehp.10334

Ateia, M., Alsbaiee, A., Karanfil, T., and Dichtel, W. (2019). Efficient PFAS Removal by Amine-Functionalized Sorbents: Critical Review of the Current Literature. Environ. Sci. Technol. Lett. 6 (12), 688-695.

Ateia, M., Arifuzzaman, M., Pellizzeri, S., Attia, M. F., Tharayil, N., Anker, J. N., et al. (2019). Cationic Polymer for Selective Removal of GenX and Short-Chain PFAS from Surface Waters and Wastewaters at Ng/L Levels. Water Res. 163, 114874. doi:10.1016/j.watres.2019.114874

Bach, C. C., Bech, B. H., Brix, N., Nohr, E. A., Bonde, J. P. E., and Henriksen, T. B. (2014). Perfluoroalkyl and Polyfluoroalkyl Substances and Human Fetal Growth: A Systematic Review. Crit. Rev. Toxicol. 45 (1), 53-67. doi:10.3109/ 10408444.2014.952400

Barrett, E. S. C. C., Chen, C., Thurston, S. W., Haug, L. S., Sabaredzovic, A., Fjeldheim, F. N., et al. (2015). Perfluoroalkyl Substances and Ovarian Hormone Concentrations in Naturally Cycling Women. Fertil. Steril. 103, 1261-1270. doi:10.1016/j.fertnstert.2015.02.001

Behr, A.-C., Lichtenstein, D., Braeuning, A., Lampen, A., and Buhrke, T. (2018). Perfluoroalkylated Substances (PFAS) Affect Neither Estrogen and Androgen Receptor Activity Nor Steroidogenesis in Human Cells In Vitro. Toxicol. Lett. 291, 51-60. doi:10.1016/j.toxlet.2018.03.029

Benninghoff, A. D., Bisson, W. H., Koch, D. C., Ehresman, D. J., Kolluri, S. K., and Williams, D. E. (2010). Estrogen-Like Activity of Perfluoroalkyl Acids In Vivo and Interaction with Human and Rainbow Trout Estrogen Receptors In Vitro. Toxi. Sci. 120 (1), 42-58. doi:10.1093/toxsci/kfq379

Birru, R. L., Liang, H.-W., Farooq, F., Bedi, M., Feghali, M., Haggerty, C. L., et al. (2021). A Pathway Level Analysis of PFAS Exposure and Risk of Gestational Diabetes Mellitus. Envi. Hea. 20 (1), 63. doi:10.1186/s12940-021-00740-z

Blake, B. E., Cope, H. A., Hall, S. M., Keys, R. D., Mahler, B. W., McCord, J., et al. (2020). Evaluation of Maternal, Embryo, and Placental Effects in CD-1 Mice Following Gestational Exposure to Perfluorooctanoic Acid (PFOA) or Hexafluoropropylene Oxide Dimer Acid (HFPO-DA or GenX). Environ. Health Perspect. 128, 027006. doi:10.1289/ehp6233

Brase, R. A., Mullin, E. J., and Spink, D. C. (2021). Legacy and Emerging Per- and Polyfluoroalkyl Substances: Analytical Techniques, Environmental Fate, and Health Effects. Int. J. Mol. Sci. 22, 995. doi:10.3390/ijms22030995

Brendel, S., Fetter, É., Staude, C., Vierke, L., and Biegel-Engler, A. (2018). Shortchain Perfluoroalkyl Acids: Environmental Concerns and a Regulatory Strategy under REACH. Environ. Sci. Eur. 30 (1), 9. doi:10.1186/s12302-018-0134-4

Cheng, W., Doering, J. A., LaLone, C., and Ng, C. (2021). Integrative Computational Approaches to Inform Relative Bioaccumulation Potential of Per- and Polyfluoroalkyl Substances across Species. Toxi. Sci. 180 (2), 212-223. doi:10.1093/toxsci/kfab004

Clara, M., Gans, O., Sanz-Escribano, D., Scharf, S., and Scheffknecht, C. (2009). Perfluorinated Alkylated Substances in the Aquatic Environment: an Austrian Case Study. Water Res. 43 (18), 4760-4768. doi:10.1016/j.watres.2009.08.004

Conley, J. M., Lambright, C. S., Evans, N., Strynar, M. J., McCord, J., McIntyre, B. S., et al. (2019). Adverse Maternal, Fetal, and Postnatal Effects of Hexafluoropropylene Oxide Dimer Acid (GenX) from Oral Gestational Exposure in Sprague-Dawley Rats. Environ. Health Perspect. 127, 37008. doi:10.1289/EHP4372

Conley, J. M., Lambright, C. S., Evans, N., McCord, J., Strynar, M. J., Hill, D., et al. (2021). Hexafluoropropylene Oxide-Dimer Acid (HFPO-DA or GenX) Alters Maternal and Fetal Glucose and Lipid Metabolism and Produces Neonatal Mortality, Low Birthweight, and Hepatomegaly in the Sprague-Dawley Rat. Environ. Int. 146, 106204. doi:10.1016/j.envint.2020.106204

Cousins, I. T., DeWitt, J. C., Glüge, J., Goldenman, G., Herzke, D., Lohmann, R., et al. (2020). Strategies for Grouping Per- and Polyfluoroalkyl Substances (PFAS) to Protect Human and Environmental Health. Environ. Sci. Process. Impacts 22 (7), 1444-1460. doi:10.1039/d0em00147c

Dalahmeh, S., Tirgani, S., Komakech, A. J., Niwagaba, C. B., and Ahrens, L. (2018). Per-and Polyfluoroalkyl Substances (PFASs) in Water, Soil and Plants in Wetlands and Agricultural Areas in Kampala, Uganda. Sci. Total Environ. 631, 660-667.
De Silva, A. O., Armitage, J. M., Bruton, T. A., Dassuncao, C., Heiger-Bernays, W., Hu, X. C., et al. (2021). PFAS Exposure Pathways for Humans and Wildlife: A Synthesis of Current Knowledge and Key Gaps in Understanding. Environ. Toxicol. Chem. 40, 631-657. doi:10.1002/etc.4935

Du, Z., Deng, S., Liu, D., Yao, X., Wang, Y., Lu, X., et al. (2016). Efficient Adsorption of PFOS and F53B from Chrome Plating Wastewater and Their Subsequent Degradation in the Regeneration Process. Chem. Eng. J. 290, 405-413. doi:10.1016/j.cej.2016.01.077

Fenton, S. E. D. A., Ducatman, A., Boobis, A., DeWitt, J. C., Lau, C., Ng, C., et al. (2021). Per- and Polyfluoroalkyl Substance Toxicity and Human Health Review: Current State of Knowledge and Strategies for Informing Future Research. Environ. Toxicol. Chem. 40 (3), 606-630. doi:10.1002/etc.4890

Fromme, H., Wöckner, M., Roscher, E., and Völkel, W. (2017). ADONA and Perfluoroalkylated Substances in Plasma Samples of German Blood Donors Living in South Germany. Int. J. Hyg. Environ. Health 220 (2), 455-460. doi:10.1016/j.ijheh.2016.12.014

Gaballah, S., Swank, A., Sobus, J. R., Howey, X. M., Schmid, J., Catron, T., et al. (2020). Evaluation of Developmental Toxicity, Developmental Neurotoxicity, and Tissue Dose in Zebrafish Exposed to GenX and Other PFAS. Environ. Health Perspect. 128 (4), 47005. doi:10.1289/EHP5843

Gebbink, W. A., van Asseldonk, L., and van Leeuwen, S. P. J. (2017). Presence of Emerging Per- and Polyfluoroalkyl Substances (PFASs) in River and Drinking Water Near a Fluorochemical Production Plant in the Netherlands. Environ. Sci. Technol. 51 (19), 11057-11065. doi:10.1021/acs.est.7b02488

Gebbink, W. A., and van Leeuwen, S. P. (2020). Environmental Contamination and Human Exposure to PFASs Near a Fluorochemical Production Plant: Review of Historic and Current PFOA and GenX Contamination in the Netherlands. Environ. Int. 137, 105583.

Giesy, J. P., and Kannan, K. (2001). Global Distribution of Perfluorooctane Sulfonate in Wildlife. Environ. Sci. Technol. 35 (7), 1339-1342. doi:10.1021/ es $001834 \mathrm{k}$

Gomis, M. I., Vestergren, R., Borg, D., and Cousins, I. T. (2018). Comparing the Toxic Potency In Vivo of Long-Chain Perfluoroalkyl Acids and Fluorinated Alternatives. Environ. Int. 113, 1-9. doi:10.1016/j.envint.2018.01.011

Han, J., and Fang, Z. (2010). Estrogenic Effects, Reproductive Impairment and Developmental Toxicity in Ovoviparous Swordtail Fish (Xiphophorus Helleri) Exposed to Perfluorooctane Sulfonate (PFOS). Aquat. Toxicol. 99 (2), 281-290. doi:10.1016/j.aquatox.2010.05.010

Hopkins, Z. R., Sun, M., DeWitt, J. C., and Knappe, D. R. U. (2018). Recently Detected Drinking Water Contaminants: GenX and Other Per- and Polyfluoroalkyl Ether Acids. J. Am. Water Works Assoc. 110 (7), 13-28. doi:10.1002/awwa.1073

Houde, M., Czub, G., Small, J. M., Backus, S., Wang, X., Alaee, M., et al. (2008). Fractionation and Bioaccumulation of Perfluorooctane Sulfonate (PFOS) Isomers in a Lake Ontario Food Web. Environ. Sci. Technol. 42 (24), 9397-9403. doi:10.1021/es800906r

Huang, S., and Jaffé, P. R. (2019). Defluorination of Perfluorooctanoic Acid (PFOA) and Perfluorooctane Sulfonate (PFOS) by Acidimicrobium Sp. Strain A6. Environ. Sci. Technol. 53 (19), 11410-11419. doi:10.1021/acs.est.9b04047

Jensen, A. A., and Leffers, H. (2008). Emerging Endocrine Disrupters: Perfluoroalkylated Substances. Int. J. Androl. 31 (2), 161-169. doi:10.1111/ j.1365-2605.2008.00870.x

Johnson, P. I., Sutton, P., Atchley, D. S., Koustas, E., Lam, J., Sen, S., et al. (2014). The Navigation Guide-Evidence-Based Medicine Meets Environmental Health: Systematic Review of Human Evidence for PFOA Effects on Fetal Growth. Environ. Health Perspect. 122 (10), 1028-1039. doi:10.1289/ehp.1307893

Kang, J. S., Choi, J.-S., and Park, J.-W. (2016). Transcriptional Changes in Steroidogenesis by Perfluoroalkyl Acids (PFOA and PFOS) Regulate the Synthesis of Sex Hormones in H295R Cells. Chemosphere 155, 436-443. doi:10.1016/j.chemosphere.2016.04.070

Kar, S., Sepúlveda, M. S., Roy, K., and Leszczynski, J. (2017). Endocrine-disrupting Activity of Per- and Polyfluoroalkyl Substances: Exploring Combined Approaches of Ligand and Structure Based Modeling. Chemosphere 184, 514-523. doi:10.1016/j.chemosphere.2017.06.024

Kelly, B. C., Ikonomou, M. G., Blair, J. D., Surridge, B., Hoover, D., Grace, R., et al. (2009). Perfluoroalkyl Contaminants in an Arctic Marine Food Web: Trophic Magnification and Wildlife Exposure. Environ. Sci. Technol. 43 (11), 4037-4043. doi:10.1021/es9003894 
Kjeldsen, L. S., and Bonefeld-Jørgensen, E. C. (2013). Perfluorinated Compounds Affect the Function of Sex Hormone Receptors. Environ. Sci. Pollut. Res. 20 (11), 8031-8044. doi:10.1007/s11356-013-1753-3

Lau, C. (2012). "Perfluorinated Compounds," in Molecular, Clinical and Environmental Toxicology. Editor A. Luch (Springer, Basel) (Experi. Suppl.), 101. doi:10.1007/978-3-7643-8340-4_3

Li, F., Duan, J., Tian, S., Ji, H., Zhu, Y., Wei, Z., et al. (2020). Short-Chain Per- and Polyfluoroalkyl Substances in Aquatic Systems: Occurrence, Impacts and Treatment. Chem. Engineer. Journ. 380, 122506. doi:10.1016/j.cej.2019.122506

Luo, K., Liu, X., Nian, M., Wang, Y., Qiu, J., Yu, H., Chen, X., et al. (2021). Environmental Exposure to Per- and Polyfluoroalkyl Substances Mixture and Male Reproductive Hormones. Envir. Intern. 152, 106496. doi:10.1016/ j.envint.2021.106496

McCarthy, C., Kappleman, W., and DiGuiseppi, W. (2017). Ecological Considerations of Per- and Polyfluoroalkyl Substances (PFAS). Curr. Pollut. Rep. 3 (4), 289-301. doi:10.1007/s40726-017-0070-8

Ateia, M., Maroli, A., Tharayil, N., and Karanfil, T. (2019). The Overlooked Shortand Ultrashort-Chain Poly- and Perfluorinated Substances: A Review. Chemosphere 220, 866-882. doi:10.1016/j.chemosphere.2018.12.186

Mokra, K. (2021). Endocrine Disruptor Potential of Short- and Long-Chain Perfluoroalkyl Substances (PFASs) -A Synthesis of Current Knowledge with Proposal of Molecular Mechanism. Intern. Journ. Mol. Scie. 22 (4), 2148. doi:10.3390/ijms22042148

Möller, A., Ahrens, L., Westerveld, J., van der Wielen, F., Ebinghaus, R., et al. (2010). Distribution and Sources of Polyfluoroalkyl Substances (PFAS) in the River Rhine Watershed. Environ. Pollut. 158 (10), 3243-3250. doi:10.1016/ j.envpol.2010.07.019

Munoz, G., Liu, J., Vo Duy, S., and Sauvé, S. (2019). Analysis of F-53B, Gen-X, ADONA, and Emerging Fluoroalkylether Substances in Environmental and Biomonitoring Samples: A Review. Tre. Enviro. Analy. Chem. 23, e00066. doi:10.1016/j.teac.2019.e00066

Nakayama, S., Strynar, M. J., Egeghy, P., Ye, X., and Lindstrom, A. B. (2007). Perfluorinated Compounds in the Cape Fear Drainage basin in North Carolina. Environ. Sci. Technol. 41 (15), 5271-5276. doi:10.1021/es070792y

Neagu, M., Constantin, C., Bardi, G., and Duraes, L. (2021). Adverse Outcome Pathway in Immunotoxicity of Perfluoroalkyls. Curr. Opin. Toxicol. 25, 23-29. doi:10.1016/j.cotox.2021.02.001

Nian, M., Luo, K., Luo, F., Aimuzi, R., Huo, X., Chen, Q., et al. (2020). Association between Prenatal Exposure to PFAS and Fetal Sex Hormones: Are the ShortChain PFAS Safer. Environ. Sci. Technol. 54 (1), 8291-8299. doi:10.1021/ acs.est.0c02444

Olsen, G. W., Gilliland, F. D., Burlew, M. M., Burris, J. M., Mandel, J. S., and Mandel, J. H. (1998). An Epidemiologic Investigation of Reproductive Hormones in Men with Occupational Exposure to Perfluorooctanoic Acid. J. Occup. Environ. Med. 40 (7), 614-622. doi:10.1097/00043764-199807000-00006

Olsen, G. W., Mair, D. C., Lange, C. C., Harrington, L. M., Church, T. R., Goldberg, C. L., et al. (2017). Per- and Polyfluoroalkyl Substances (PFAS) in American Red Cross Adult Blood Donors, 2000-2015. Environ. Res. 157, 87-95. doi:10.1016/j.envres.2017.05.013

Pan, Y., Zhang, H., Cui, Q., Sheng, N., Yeung, L. W. Y., Sun, Y., et al. (2018). Worldwide Distribution of Novel Perfluoroether Carboxylic and Sulfonic Acids in Surface Water. Environ. Sci. Technol. 52 (14), 7621-7629. doi:10.1021/ acs.est.8b00829

Petersen, K. U., Larsen, J. R., Deen, L., Flachs, E. M., Hærvig, K. K., Hull, S. D., et al. (2020). Per-and Polyfluoroalkyl Substances and Male Reproductive Health: A Systematic Review of the Epidemiological Evidence. J. Toxicol. Environ. Health B 23 (6), 276-291.

Petersen, M., Halling, J., Jørgensen, N., Nielsen, F., Grandjean, P., Jensen, T., et al. (2018). Reproductive Function in a Population of Young Faroese Men with Elevated Exposure to Polychlorinated Biphenyls (PCBs) and Perfluorinated Alkylate Substances (PFAS). Int. J. Environ. Res. Public Health 15, 1880. doi:10.3390/ijerph15091880

Qu, J.-H., Lu, C.-C., Xu, C., Chen, G., Qiu, L.-L., Jiang, J.-K., et al. (2016). Perfluorooctane Sulfonate-Induced Testicular Toxicity and Differential Testicular Expression of Estrogen Receptor in Male Mice. Environ. Toxicol. Pharmacol. 45, 150-157. doi:10.1016/j.etap.2016.05.025

Sakr, C. J., Kreckmann, K. H., Green, J. W., Gillies, P. J., Reynolds, J. L., and Leonard, R. C. (2007). Cross-Sectional Study of Lipids and Liver Enzymes
Related to a Serum Biomarker of Exposure (Ammonium Perfluorooctanoate or APFO) as Part of a General Health Survey in a Cohort of Occupationally Exposed Workers. Journ. Occu. Enviro. Med. 49 (10), 1086-1096. doi:10.1097/ jom.0b013e318156eca3

Shi, X., Du, Y., Lam, P. K. S., Wu, R. S. S., and Zhou, B. (2008). Developmental Toxicity and Alteration of Gene Expression in Zebrafish Embryos Exposed to PFOS. Toxicol. Appl. Pharmacol. 230 (1), 23-32. doi:10.1016/j.taap.2008.01.043 Shi, G., Cui, Q., Pan, Y., Sheng, N., Sun, S., Guo, Y., et al. (2017). 6:2 Chlorinated Polyfluorinated Ether Sulfonate, a PFOS Alternative, Induces Embryotoxicity and Disrupts Cardiac Development in Zebrafish Embryos. Aquat. Toxicol. 185, 67-75. doi:10.1016/j.aquatox.2017.02.002

Shi, G., Wang, J., Guo, H., Sheng, N., Cui, Q., Pan, Y., et al. (2019). Parental Exposure to 6:2 Chlorinated Polyfluorinated Ether Sulfonate (F-53B) Induced Transgenerational Thyroid Hormone Disruption in Zebrafish. Sci. Total Environ. 665, 855-863. doi:10.1016/j.scitotenv.2019.02.198

Sinclair, G. M., Long, S. M., and Jones, O. A. H. (2020). What Are the Effects of PFAS Exposure at Environmentally Relevant Concentrations. Chemosphere 258, 127340. doi:10.1016/j.chemosphere.2020.127340

So, M. K., Miyake, W. Y., Ho, Y. M., Taniyasu, S., Rostkowski, P., et al. (2007). Perfluorinated Compounds in the Pearl River and Yangtze River of China. Chemosphere 68 (11), 2085-2095. doi:10.1016/j.chemosphere.2007.02.008

Sunderland, E. M., Hu, X. C., Dassuncao, C., Tokranov, A. K., Wagner, C. C., and Allen, J. G. (2019). A Review of the Pathways of Human Exposure to Poly- and Perfluoroalkyl Substances (PFASs) and Present Understanding of Health Effects. J. Expo. Sci. Environ. Epidemiol. 29 (2), 131-147. doi:10.1038/s41370-018-0094-1

Tao, L., Ma, J., Kunisue, T., Libelo, E. L., Tanabe, S., and Kannan, K. (2008). Perfluorinated Compounds in Human Breast Milk from Several Asian Countries, and in Infant Formula and Dairy Milk from the United States. Environ. Sci. Technol. 42 (22), 8597-8602. doi:10.1021/es801875v

Tarapore, P., and Ouyang, B. (2021). Perfluoroalkyl Chemicals and Male Reproductive Health: Do PFOA and PFOS Increase Risk for Male Infertility. Ijerph 18 (7), 3794. doi:10.3390/ijerph18073794

Taylor, K. W., Hoffman, K., Thayer, K. A., and Daniels, J. L. (2014). Polyfluoroalkyl Chemicals and Menopause Among Women 20-65 Years of Age (NHANES). Environ. Health Perspect. 122, 145-150. doi:10.1289/ ehp. 1306707

Tsai, M.-S., Lin, C.-Y., Lin, C.-C., Chen, M.-H., Hsu, S. H. J., Chien, K.-L., et al. (2015). Association between Perfluoroalkyl Substances and Reproductive Hormones in Adolescents and Young Adults. Int. J. Hyg. Environ. Health 218 (5), 437-443. doi:10.1016/j.ijheh.2015.03.008

Tsuda, S. (2016). Differential Toxicity between Perfluorooctane Sulfonate (PFOS) and Perfluorooctanoic Acid (PFOA). Journ. Toxico. Scie. 41, SP27-SP36. doi:10.2131/jts.41.sp27

USEPA (2018). Technical Fact Sheet: Draft ToxicityAssessmentsfor GenX Chemicals and PFBS. Available at: https://www.epa.gov/sites/production/ files/2018-12/documents/tech_fact_sheet_genx_pfbs_draft_tox_assess_final_ 508.pdf (Accessed July 19, 2021).

USEPA (United States Environmental Protection Agency) (2021). Contaminant Candidate List (CCL) and Regulatory Determination 4. Available at: https:// www.epa.gov/ccl/regulatory-determination-4 (Accessed August 4, 2021).

Vestergren, R., and Cousins, I. T. (2009). Tracking the Pathways of Human Exposure to Perfluorocarboxylates. Environ. Sci. Technol. 43 (15), 5565-5575. doi:10.1021/es900228k

Vitti, M. D. E. G., Di Emidio, G., Di Carlo, M., Carta, G., Antonosante, A., Artini, P. G., et al. (2016). Peroxisome Proliferator-Activated Receptors in Female Reproduction and Fertility. PPAR Res. 2016, 1-12. doi:10.1155/ $2016 / 4612306$

Wang, Y., Shi, Y., and Cai, Y. (2019). Spatial Distribution, Seasonal Variation and Risks of Legacy and Emerging Per- and Polyfluoroalkyl Substances in Urban Surface Water in Beijing, China. Sci. Total Environ. 673, 177-183. doi:10.1016/ j.scitotenv.2019.04.067

Wang, Z., DeWitt, J. C., Higgins, C. P., and Cousins, I. T. (2017). A Never-Ending Story of Per- and Polyfluoroalkyl Substances (PFASs). Environ. Sci. Technol. 51 (5), 2508-2518. doi:10.1021/acs.est.6b04806

Wang, X., Bai, Y., Tang, C., Cao, X., Chang, F., and Chen, L. (2018). Impact of Perfluorooctane Sulfonate on Reproductive Ability of Female Mice through Suppression of Estrogen Receptor a-Activated Kisspeptin Neurons. Toxico. Scie. 165 (2), 475-486. doi:10.1093/toxsci/kfy167 
Wang, Z., Zhang, T., Wu, J., Wei, X., Xu, A., Wang, S., et al. (2021). Male Reproductive Toxicity of Perfluorooctanoate (PFOA): Rodent Studies. Chemo. 270, 128608. doi:10.1016/j.chemosphere.2020.128608

Xie, X., Weng, X., Liu, S., Chen, J., Guo, X., Gao, X., et al. (2021). Perfluoroalkyl and Polyfluoroalkyl Substance Exposure and Association with Sex Hormone Concentrations: Results from the NHANES 2015-2016. Environ. Sci. Eur. 33, 69. doi:10.1186/s12302-021-00508-9

Xuelian Bai, Y. S. (2021). Perfluoroalkyl Substances (PFAS) in Surface Water and Sediments from Two Urban Watersheds in Nevada. USA. Scie. Tot. Enviro. 751, 141622. doi:10.1016/j.scitotenv.2020.141622

Yahia, D., El-Nasser, M. A., Abedel-Latif, M., Tsukuba, C., Yoshida, M., Sato, I., et al. (2010). Effects of Perfluorooctanoic Acid (PFOA) Exposure to Pregnant Mice on Reproduction. J. Toxicol. Sci. 35 (4), 527-533. doi:10.2131/jts.35.527

Yao, P.-L., Ehresman, D. J., Rae, J. M. C., Chang, S.-C., Frame, S. R., Butenhoff, J. L., et al. (2014). Comparative In Vivo and In Vitro Analysis of Possible Estrogenic Effects of Perfluorooctanoic Acid. Toxicology 326, 62-73. doi:10.1016/ j.tox.2014.10.008

Zeng, Z., Song, B., Xiao, R., Zeng, G., Gong, J., Chen, M., et al. (2019). Assessing the Human Health Risks of Perfluorooctane Sulfonate by In Vivo and In Vitro Studies. Environ. Int. 126, 598-610. doi:10.1016/j.envint.2019.03.002

Zhang, N., Wang, W. S., Li, W. J., Liu, C., Wang, Y., and Sun, K. (2015). Reduction of Progesterone, Estradiol and hCG Secretion by Perfluorooctane Sulfonate via Induction of Apoptosis in Human Placental Syncytiotrophoblasts. Placenta 36 (5), 575-580. doi:10.1016/j.placenta.2015.02.008

Zhang, X., Lohmann, R., Dassuncao, C., Hu, X. C., Weber, A. K., Vecitis, C. D., et al. (2016). Source Attribution of Poly-And Perfluoroalkyl Substances (PFASs) in Surface Waters from Rhode Island and the New York Metropolitan Area. Placenta 3 (9). doi:10.1016/j.placenta.2015.02.008

Zhao, Y., TanZhao, Y. S., Strynar, M. J., Strynar, M. J., Perez, G., Haslam, S. Z., et al. (2012). Perfluorooctanoic Acid Effects on Ovaries Mediate its Inhibition of Peripubertal Mammary Gland Development in Balb/c and C57Bl/6 Mice. Reprod. Toxicol. 33 (4), 563-576. doi:10.1016/j.reprotox.2012.02.004

Zhao, P., Xia, X., Dong, J., Xia, N., Jiang, X., Li, Y., et al. (2016). Short- and LongChain Perfluoroalkyl Substances in the Water, Suspended Particulate Matter, and Surface Sediment of a Turbid River. Sci. Total Environ. 568, 57-65. doi:10.1016/j.scitotenv.2016.05.221

Zheng, X.-M., Liu, H.-L., Shi, W., Wei, S., Giesy, J. P., and Yu, H.-X. (2012). Effects of Perfluorinated Compounds on Development of Zebrafish Embryos. Environ. Sci. Pollut. Res. 19 (7), 2498-2505. doi:10.1007/s11356-012-0977-y

Zhou, Z., Liang, Y., Shi, Y., Xu, L., and Cai, Y. (2013). Occurrence and Transport of Perfluoroalkyl Acids (PFAAs), Including Short-Chain PFAAs in Tangxun Lake, China. Environ. Sci. Technol. 47 (16), 9249-9257. doi:10.1021/es402120y

Zhou, W., Zhang, L., Tong, C., Fang, F., Zhao, S., Tian, Y., et al. (2017). Plasma Perfluoroalkyl and Polyfluoroalkyl Substances Concentration and Menstrual Cycle Characteristics in Preconception Women. Enviro. Hea. Persp. 125, 067012. doi:10.1289/ehp1203

Zhou, Y., Wang, T., Jiang, Z., Kong, X., Li, Q., Sun, Y., et al. (2017). Ecological Effect and Risk towards Aquatic Plants Induced by Perfluoroalkyl Substances: Bridging Natural to Culturing Flora. Chemosphere 167, 98-106. doi:10.1016/ j.chemosphere.2016.09.146

Conflict of Interest: The authors declare that the research was conducted in the absence of any commercial or financial relationships that could be construed as a potential conflict of interest.

Publisher's Note: All claims expressed in this article are solely those of the authors and do not necessarily represent those of their affiliated organizations, or those of the publisher, the editors and the reviewers. Any product that may be evaluated in this article, or claim that may be made by its manufacturer, is not guaranteed or endorsed by the publisher.

Copyright (c) 2021 Chambers, Hopkins and Richards. This is an open-access article distributed under the terms of the Creative Commons Attribution License (CC BY). The use, distribution or reproduction in other forums is permitted, provided the original author(s) and the copyright owner(s) are credited and that the original publication in this journal is cited, in accordance with accepted academic practice. No use, distribution or reproduction is permitted which does not comply with these terms. 